\title{
Finite-size scaling of entanglement entropy at the Anderson transition with interactions
}

\author{
An Zhao, ${ }^{1}$ Rui-Lin Chu, ${ }^{1,2}$ and Shun-Qing Shen ${ }^{1}$ \\ ${ }^{1}$ Department of Physics, University of Hong Kong, Pokfulam Road, Hong Kong, China \\ ${ }^{2}$ Department of Physics, University of Texas at Dallas, Richardson, Texas 75080, USA
}

(Received 9 October 2012; published 30 May 2013)

\begin{abstract}
We study the Anderson transition with interactions in one dimension from the perspective of quantum entanglement. Extensive numerical calculations of the entanglement entropy (EE) of the systems are carried out through the density matrix renormalization group algorithm. We demonstrate that the EE can be used for the finite-size scaling (FSS) to characterize the Anderson transition in both noninteracting and interacting systems. From the FSS analysis we can obtain a precise estimate of the critical parameters of the transition. The method can be applied to various one-dimensional models, either interacting or noninteracting, to quantitatively characterize the Anderson transitions.
\end{abstract}

DOI: 10.1103/PhysRevB.87.205140

PACS number(s): 73.20.Fz, 03.65.Ud, 71.30.+h, 72.15.Rn

\section{INTRODUCTION}

In the theory of Anderson localization, all states are localized by disorder in low dimensions when interactions are absent. ${ }^{1-3}$ Theories indeed predict that strong attractive interactions are able to induce a delocalized phase..$^{4,5}$ Despite that various widely accepted numerical models of Anderson transitions have neglected interactions, recent studies do suggest that inclusion of interactions is necessary when explaining the experiments. ${ }^{6}$ However, since including the interactions makes the model a many-body system, numerical investigations are computationally challenging. There has been only very limited success in numerical simulations of Anderson transitions with interactions. ${ }^{7-9}$ Although numerical methods for studying Anderson transitions of single-particle systems have developed into standard procedures, ${ }^{6,10-12}$ alternative methods must be sought when interactions are involved since only very limited system sizes can be numerically calculated. Schmitteckert et al. and Schuster et al. investigated the system's phase sensitivity of the ground-state energy to roughly estimate the delocalized phase, ${ }^{7,8}$ while Cater and Mackinnon tried to calculate the localization length directly with the transfer-matrix method. ${ }^{9}$

For the single-particle systems, the finite-size scaling (FSS) method provides the most precise quantitative characterization of the Anderson transition. ${ }^{6,12}$ A handful of physical quantities can be used for FSS, such as localization length, conductance, density of states, and topological numbers. ${ }^{11}$ However, for the many-body systems, such quantitative characterization of the Anderson transition has been lacking.

In this paper we demonstrate that the quantum entanglement entropy (EE) can be adopted as a FSS quantity for Anderson transitions in one dimension. By carrying out the scaling analysis in the framework of one-parameter scaling theory, we give an estimate for various critical parameters of the transition. Before being applied to the interacting system, the method is first tested in the noninteracting system, where the result is consistent with that given by the wellestablished transfer-matrix method.

\section{QUANTUM ENTANGLEMENT ENTROPY}

The $\mathrm{EE}$ is a measure of the quantum correlations in a system. ${ }^{13}$ For a pure state $|\Psi\rangle$ of bipartite system AB, the
EE (von Neumann entropy) is defined as

$$
S=-\operatorname{Tr} \rho_{A} \log \rho_{A}=-\operatorname{Tr} \rho_{B} \log \rho_{B},
$$

where $\rho_{A(B)}=\operatorname{Tr}_{B(A)}|\Psi\rangle\langle\Psi|$ is the reduced density matrix of subsystem A(B). Studies have shown that it can be used to characterize both quantum criticality and topological phases in a variety of quantum many-body systems. ${ }^{14-20}$ Recently there has also been increasing interest in characterizing the disordered quantum systems through the EE. ${ }^{21-26}$

Since the amount of correlation is restricted by the system's correlation length, localization is naturally manifested in the EE. Consider a one-dimensional (1D) gapless system of length $L$ and partitioned into two halves; in the clean limit as well as in the delocalized phase, the EE shows logarithmic divergence $S_{H} \sim(c / 6) \log L$, where $c$ is a universal constant given by the central charge of the associated conformal field theory. For localized system, when $L \rightarrow \infty$, the EE only saturates to $S_{H} \sim(c / 6) \log \xi$, where $\xi$ is the localization length. ${ }^{15}$ When the system is finite, behavior of EE is affected by $L$ and becomes subtle. To study the $\xi$ in a localized finite system, Berkovits proposed a heuristic scaling function of EE, which describes the crossover from the logarithmical behavior of EE to the saturated one. ${ }^{27}$ However, in the critical regime where $\xi$ is divergent and hence much larger than the system size $L$, the saturation of EE is mainly caused by the finite $L$ and the influence of $\xi$ becomes minute. It is difficult to extract the value of $\xi$ with any confidence in this case. Thus we resort to FFS of EE and aim to find out the effect of $\xi$.

\section{MODEL HAMILTONIAN}

We start by describing our model Hamiltonian, which is a 1D spinless fermion wire of length $L$ with attractive nearest neighbor (NN) interactions

$$
\begin{aligned}
\mathcal{H}= & -t \sum_{i=1}^{L-1}\left(c_{i}^{\dagger} c_{i+1}+\text { H.c. }\right)+\sum_{i=1}^{L} \epsilon_{i} c_{i}^{\dagger} c_{i} \\
& +U \sum_{i=1}^{L-1}\left(n_{i}-1 / 2\right)\left(n_{i+1}-1 / 2\right)-\mu \sum_{i=1}^{L} c_{i}^{\dagger} c_{i},
\end{aligned}
$$

where $c_{i}$ and $c_{i}^{\dagger}$ are the annihilation and creation operators, $n_{i}=c_{i}^{\dagger} c_{i}$. The first two terms constitute the standard Anderson 
model while the third term represents the attractive $\mathrm{NN}$ interaction $(U<0)$. The disorder is introduced as random on-site potentials $\epsilon_{i}$ with a uniform distribution in the range $[-W / 2, W / 2]$. Hopping amplitude is set to $t=1$. The last term represents the chemical potential which controls the particle density of the system. Disorder has no net contribution to the chemical potential since we tune $\sum_{i=1}^{L} \epsilon_{i}=0$. Because the total number of particles is conserved in this system, we will restrict ourselves to the fixed total particle number at half filling. Also zero temperature is assumed.

In the clean limit, up to a Jordan-Wigner transformation, this model is equivalent to the $S=1 / 2 X X Z$ spin-chain model, for which there exists the Bethe-ansatz solution that can be used to guide our numerical simulation. For $|U|<$ 2 , the system is gapless (i.e., metallic). For $U>2$, the charge-density-wave (CDW) phase renders the system a Mott insulator; for $U<-2$, the ground state becomes unstable to phase separation, being either empty or full filling. ${ }^{28}$ For the disordered case, an insulator to metal transition is predicted at $U \approx-1 .^{4,5}$ A second metal to insulator transition happens at further stronger interaction, where the fermions start to form clusters and become localized again. In this paper, we set our scope in the range $-2<U<0$.

The EE is numerically obtained with the density matrix renormalization group (DMRG) algorithm, which allows the calculation of ground state and relevant physical quantities for large interacting systems with high accuracy. ${ }^{29,30}$ In our case, we consider open wires of variant sizes up to $L=1024$. During the DMRG calculation, we retained 250 (for $L<1024$ ) and 330 (for $L=1024$ ) states for each block, and performed lattice sweeping until both the ground-state energy and the EE are converged.

\section{NONINTERACTING CASE}

In the special case when $U=0$ the model recovers to a single-particle system. In such case no true Anderson transition exists in the system as the only delocalized point is the clear limit where $W=0$. However, since it is well established that the localization length in such a system shows power-law divergence as $\xi \approx 105 / W^{2}$ close to the clean limit (i.e., with a "critical exponent" $v=2.0),{ }^{31}$ we can use it as a benchmark for the scaling analysis. In this case the EE can be obtained quickly by the method developed by Peschel without the bother of DMRG. ${ }^{32}$ Also averaging over a large number of random disorder configurations is allowed.

The scaling quantity adopted is $\Delta S_{H}=S_{H}-S_{H}^{\prime}$, where $S_{H}$ and $S_{H}^{\prime}$ are the EE of the clean and disordered systems, respectively. The scaling behavior is shown in Fig. 1 for the noninteracting case with system size up to $L=1024.10^{4}$ random disorder configurations have been collected for each data point of all system sizes. We then fit the dependence of $\Delta S_{H}$ on the system size $L$ and disorder $W$ to a one-parameter scaling law incorporated with a background term

$$
\Delta S_{H}=f(x)+c(U, W),
$$

where the dimensionless $x$ is defined as $x=L / 2 \xi . c(U, W)$ is the background term which is scaling irrelevant. ${ }^{6}$ Since $\xi$ is divergent in the critical regime, $x$ is vanishingly small. This
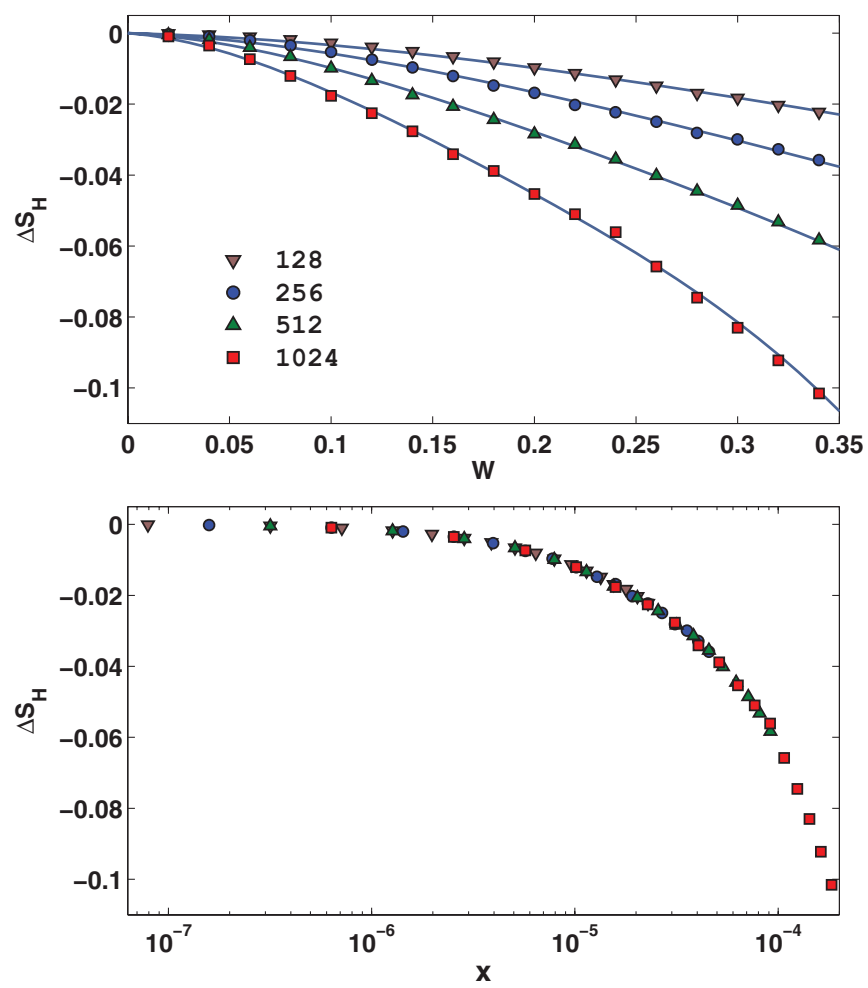

FIG. 1. (Color online) Upper panel: Scaling of $\Delta S_{H}$ for the noninteracting case against disorder strength with different system sizes; the solid lines represent the best fit of the scaling function with $n=2$. Lower panel: Data collapse to the one-parameter scaling function $f(x)$.

allows us to do a power expansion of the scaling function

$$
f(x)=x^{1 / v}+a_{2} x^{2 / v}+\cdots+a_{n} x^{n / v} .
$$

Here, $v$ is the critical exponent that characterizes the divergence of the localization length against the controlling parameter $\chi$ :

$$
\xi=\xi_{0}\left|\chi-\chi_{c}\right|^{-v}
$$

For the noninteracting case the only controlling parameter in our model is $W$ and obviously $W_{c}=0$. In Eq. (4), $a_{1}$ has been set to unity to avoid redundancy in the fitting parameters, which also means the absolute value of $\xi_{0}$ is not determined in our fitting. In the noninteracting case when $x=0$ the system is exactly clean, which means $\Delta S_{H}=0$, and thus $c(0,0)=0$. We can also do a power expansion for the background term $c(0, W)$ against $W$. But during testing we find this term is vanishingly small; thus we will treat this term as zero for this very case. This will reduce the total number of free fitting parameters to $n_{t}=n+1$. The fitting result is presented in Table I. We find it is sufficient to terminate the expansion

TABLE I. Best fit of the critical exponent with $95 \%$ confidence intervals for the noninteracting case with different number of expansion terms. $0<W<0.35$.

\begin{tabular}{cccc}
\hline \hline$n$ & 2 & 3 & 4 \\
\hline$v$ & $2.04 \pm 3 \%$ & $2.03 \pm 3 \%$ & $1.99 \pm 1.5 \%$ \\
\hline \hline
\end{tabular}


TABLE II. Same as Table I but fixing $n=4$ and using different ranges of $W$.

\begin{tabular}{lccc}
\hline \hline$W$ & $(0,0.35)$ & $(0,0.25)$ & $(0,0.15)$ \\
\hline$v$ & $1.99 \pm 1.5 \%$ & $1.99 \pm 1.5 \%$ & $2.00 \pm 1 \%$ \\
\hline \hline
\end{tabular}

terms at $n=2$ for the scaling function. Considering more expansion terms yields consistent results. We have also tested the fitting stability using different ranges of $W$; the result also shows good consistency (see Table II). The estimated "critical exponent" $v$ agrees precisely with that given by the well-established transfer-matrix method in Ref. 31.

To verify the validity of the one-parameter scaling of the EE, we need to see how the data collapse to the scaling function, which supposedly is a universal fit to the data of all systems regardless of the size $L$. This is shown in the lower panel of Fig. 1, where the data from the upper panel are replotted as a function of the dimensionless $x$. All points collapse nicely to a single curve which corresponds to a fit with $n=2$. Although the absolute value of $\xi_{0}$ (and hence $\xi$ ) is not determined in the fitting, it does not affect the data collapse since what $\xi_{0}$ does is merely shifting the curve horizontally in the plot.

\section{INTERACTING CASE}

Next we switch the interaction on and calculate the EE with the DMRG algorithm. In this case our aim is to identify the interaction-induced Anderson localization-delocalization transition. We choose a fixed disorder strength $W=1.0$ and sweep $U$. For the disordered systems the $\mathrm{EE}$ is averaged over 1000 (for $L<1024$ ) and 400 (for $L=1024$ ) random disorder configurations.

The EE of both clean (solid lines) and disordered (dotted lines) systems are plotted in Fig. 2. For the clean case the EE scales as $(1 / 6) \log L$. However, for the disordered case the $\mathrm{EE}$ is restricted by localization and hence deviates from

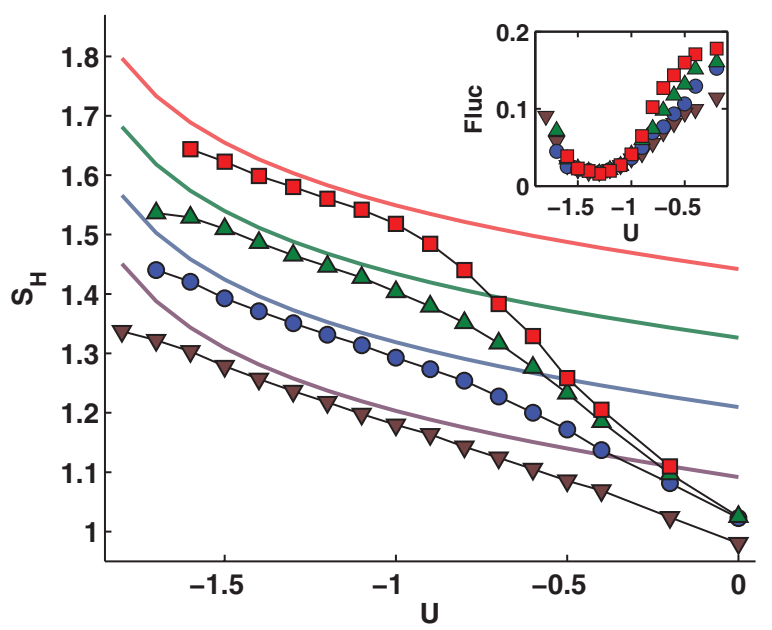

FIG. 2. (Color online) $S_{H}$ for the interacting case of both clean (solid lines) and corresponding disordered (dot lines) systems for different system sizes. The inset shows the corresponding fluctuations of $S_{H}$. In the delocalized phase, the EE's fluctuation is found to be minimum. $W=1.0$.
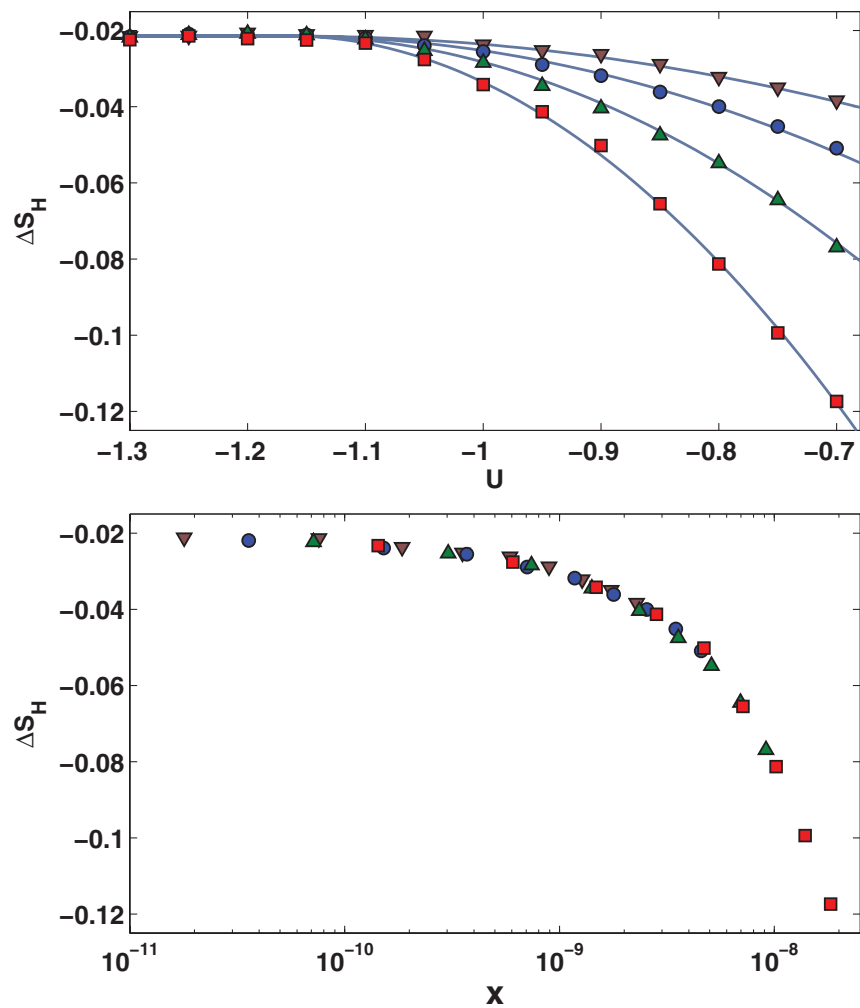

FIG. 3. (Color online) Upper panel: Scaling of $\Delta S_{H}$ against interaction with different system sizes; the solid lines represent the best fit of the scaling function with $n=2$. Lower panel: Data collapse to the one-parameter scaling function $f(x) . W=1.0$.

logarithmic scaling. But in a certain range of $U$ the EE recovers to the same logarithmic scaling as the clean case. In the weakinteraction regime, the EE's difference between the clean and disordered case is clearly dependent on both the system size and the interaction. When the interaction becomes stronger this dependence gradually disappears but finally reemerges. The same trend can also be observed in the fluctuations of the EE (inset of Fig. 2). This signatures two transitions: first from localization to delocalization and then from delocalization to localization. In the delocalized phase $\Delta S_{H}$ is only allowed to be a scaling-irrelevant quantity since both $S_{H}$ and $S_{H}^{\prime}$ scales as $(1 / 6) \log L$ in this regime. In Fig. 2, this regime locates roughly at $-1.5<U<1$. The same observation can be made when fixing $U$ and sweep $W$. As is shown in Fig. 4, in a certain range of $W$ the $\Delta S_{H}$ becomes size independent but is a function of $W$ only. This phenomena itself can be used to give a rough estimate of the delocalized phase, just as is done in Ref. 7 using the ground-state phase sensitivity.

To qualitatively characterize the phase transition, we need more extensive simulations at the vicinity of the transition point. We will fix $W$ and use $U$ as the controlling parameter in the FSS. Due to the system's instability to phase separation at strong interactions, which is exaggerated by the disorder, we find it impossible to stick to half filling in the DMRG calculations when $U<-1.8$. We thus choose to demonstrate the FSS analysis at the first Anderson transition where sufficient reliable EE data can be collected. 


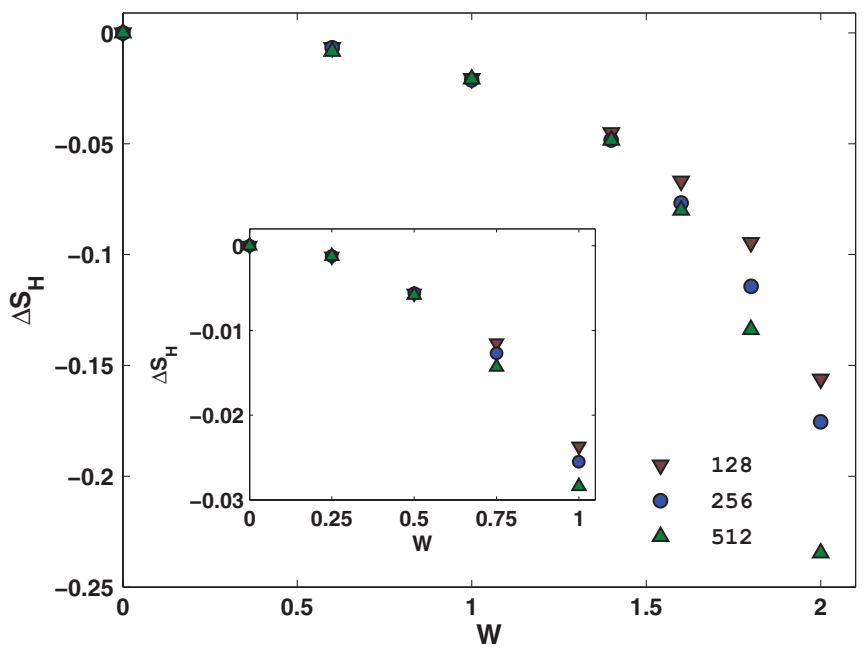

FIG. 4. (Color online) $\Delta S_{H}$ for different system sizes as a function of $W$ when fixing $U=-1.2$. Inset: $U=-1.0$.

In the upper panel of Fig. 3 we plot the scaling behavior of $\Delta S_{H}$ against $U$. The delocalized phase clearly appears as a plateau in the plot. $\Delta S_{H}$ shows almost no dependence on $U$ in the delocalized phase as is compared with Fig. 4 in which it clearly is a function of $W$. This allows us to treat the background term $c(U, 1.0)$ as a small constant $a_{0}$ in the scaling function Eq. (3). We will assume $\xi=\infty$ when $U<$ $U_{c}$. Accordingly Eq. (5) is modified as

$$
\xi^{-1}=\theta\left(U-U_{c}\right) \xi_{0}^{-1}\left(U-U_{c}\right)^{v}
$$

in the fitting, where $\theta(x)$ is the step function. In this case the total number of free fitting parameters is $n_{t}=n+3$ since both $a_{0}$ and $U_{c}$ also need to be determined by fitting. We have also considered different numbers of expansion terms for the scaling function. The result shows good consistency when using $n \geqslant 2$. As is listed in Table III, when increasing $n$ the estimated $U_{c}$ shows almost no change; only $v$ slightly varies. In the lower panel of Fig. 3, the one-parameter scaling is demonstrated graphically by replotting the data with $U>U_{c}$ as a function of the dimensionless $x$. Again the absolute value
TABLE III. Best fit of the critical parameters with $95 \%$ confidence intervals for the interacting case with different number of expansion terms. $W=1.0$.

\begin{tabular}{lrrr}
\hline \hline$n$ & \multicolumn{1}{c}{2} & \multicolumn{1}{c}{3} & \multicolumn{1}{c}{4} \\
\hline$v$ & $2.33 \pm 7 \%$ & $2.31 \pm 9 \%$ & $2.35 \pm 9 \%$ \\
$U_{c}$ & $-1.15 \pm 2 \%$ & $-1.15 \pm 3 \%$ & $-1.15 \pm 3 \%$ \\
\hline \hline
\end{tabular}

of $\xi$ is not determined but only the critical exponent $v$ that describes $\xi$ 's divergence is meaningful. The obtained $U_{c}$ agrees with the roughly estimated phase boundary of Ref. 7 . But both $U_{c}$ and $v$ deviates from Ref. 9, in which no FSS is involved and the estimated $\xi$ is limited to the maximum system size $L$ instead of diverging.

\section{CONCLUSION}

In summery, we have shown that the $\mathrm{EE}$ is an efficient quantity in characterizing the Anderson transition in one dimension. The demonstrated FSS of the EE can be used to quantitatively characterize the Anderson transitions in 1D systems. The precision of the method can be improved by collecting a large number of random disorder configurations for averaging. This is particularly feasible for the noninteracting systems where calculation of $\mathrm{EE}$ is not computationally demanding. This is evident by comparing the data collapse in our Figs. 1 and 3. Obviously the more intensive sample averaging in Fig. 1 has helped to produce better data collapse and a more precise determinant of the scaling function. For the interacting systems, since the calculation of the $\mathrm{EE}$ is naturally incorporated with the standard DMRG algorithm, the method can also be readily applied.

\section{ACKNOWLEDGMENTS}

We gratefully acknowledge discussions with Cenke $\mathrm{Xu}$ and Chuanwei Zhang. This work is supported by the Research Grant Council of Hong Kong under Grant No. HKU 705111P. R.-L.C. is also supported by AFOSR (FA9550-11-1-0313) and ARO (W911NF-09-1-0248).
${ }^{1}$ P. W. Anderson, Phys. Rev. 109, 1492 (1958).

${ }^{2}$ E. Abrahams, P. W. Anderson, D. C. Licciardello, and T. V. Ramakrishnan, Phys. Rev. Lett. 42, 673 (1979).

${ }^{3}$ P. A. Lee and T. V. Ramakrishnan, Rev. Mod. Phys. 57, 287 (1985). ${ }^{4}$ W. Apel and T. M. Rice, Phys. Rev. B 26, 7063 (1982).

${ }^{5}$ T. Giamarchi and H. J. Schulz, Phys. Rev. B 37, 325 (1988).

${ }^{6}$ K. Slevin and T. Ohtsuki, Phys. Rev. B 80, 041304(R) (2009).

${ }^{7}$ P. Schmitteckert, T. Schulze, C. Schuster, P. Schwab, and U. Eckern, Phys. Rev. Lett. 80, 560 (1998); P. Schmitteckert, R. A. Jalabert, D. Weinmann, and J. L. Pichard, ibid. 81, 2308 (1998).

${ }^{8}$ C. Schuster, R. A. Römer, and M. Schreiber, Phys. Rev. B 65, 115114 (2002).

${ }^{9}$ J. M. Carter and A. MacKinnon, Phys. Rev. B 72, 024208 (2005).
${ }^{10}$ A. MacKinnon and B. Kramer, Z. Phys. B 53, 1 (1983).

${ }^{11}$ B. Huckestein, Rev. Mod. Phys. 67, 357 (1995).

${ }^{12}$ Y. Asada, K. Slevin, and T. Ohtsuki, Phys. Rev. Lett. 89, 256601 (2002).

${ }^{13}$ For a review see L. Amico, R. Fazio, A. Osterloh, and V. Vedral, Rev. Mod. Phys. 80, 517 (2008).

${ }^{14}$ G. Vidal, J. I. Latorre, E. Rico, and A. Kitaev, Phys. Rev. Lett 90, 227902 (2003).

${ }^{15}$ P. Calabrese and J. Cardy, J. Stat. Mech. (2004) P06002.

${ }^{16}$ S. Ryu and Y. Hatsugai, Phys. Rev. B 73, 245115 (2006).

${ }^{17}$ G. Refael and J. E. Moore, Phys. Rev. Lett. 93, 260602 (2004).

${ }^{18}$ F. Pollmann and J. E. Moore, New J. Phys. 12, 025006 (2010). 
${ }^{19}$ A. M. Turner, F. Pollmann, and E. Berg, Phys. Rev. B 83, 075102 (2011).

${ }^{20}$ L. Tagliacozzo, T. R. de Oliveira, S. Iblisdir, and J. I. Latorre, Phys. Rev. B 78, 024410 (2008).

${ }^{21}$ M. Fagotti, P. Calabrese, and J. E. Moore, Phys. Rev. B 83, 045110 (2011).

${ }^{22}$ F. Igloi and Y. C. Lin, J. Stat. Mech. (2008) P06004.

${ }^{23}$ X. Jia, A. R. Subramaniam, I. A. Gruzberg, and S. Chakravarty, Phys. Rev. B 77, 014208 (2008).

${ }^{24}$ J. H. Bardarson, F. Pollmann, and J. E. Moore, Phys. Rev. Lett. 109, 017202 (2012).

${ }^{25}$ N. Laflorencie, Phys. Rev. B 72, 140408 (2005).
${ }^{26}$ R. Santachiara, J. Stat. Mech. (2006) L06002.

${ }^{27}$ R. Berkovits, Phys. Rev. Lett. 108, 176803 (2012).

${ }^{28}$ C. N. Yang and C. P. Yang, Phys. Rev. 150, 321 (1966); 150, 327 (1966).

${ }^{29}$ S. R. White, Phys. Rev. Lett. 69, 2863 (1992); S. R. White and R. M. Noack, ibid. 68, 3487 (1992); S. R. White, Phys. Rev. B 48, 10345 (1993).

${ }^{30}$ F. Pollmann, S. Mukerjee, A. M. Turner, and J. E. Moore, Phys. Rev. Lett. 102, 255701 (2009).

${ }^{31}$ R. A. Römer and M. Schreiber, Phys. Rev. Lett. 78, 515 (1997).

${ }^{32}$ I. Peschel, J. Phys. A: Math. Gen. 36, L205 (2003). 